\title{
BUSINESS SUCCESS THROUGH UNDERSTANDING HUMAN EMOTIONS: CASE STUDY OF CLASSIFYING EMOTIONS USING THE BRAIN WAIVES EEG DATA
}

\author{
Rohith Reddy Peesari ${ }^{1}$, Jinan Fiaidhi ${ }^{1}$ and Sabah Mohammed ${ }^{1}$ \\ ${ }^{1}$ Department of Computer Science, Lakehead University, Canada \\ ${ }^{1}$ \{rpeesari, jfiaidhi, mohammed\} @lakeheadu.ca
}

\begin{abstract}
Emotion analytics applied in a business setting provide solutions to understand customer emotions or employee performance and carry out real-time AI analysis of the data. This is a smarter, much more unbiased alternative to such business tools as email surveys, mystery shoppers or "rate the service" buttons practices that tend to be subjective and inconsistent and often fail to produce accurate and meaningful data. Many kinds of research are conducted to detect the emotion of a person by using different formats of data like speech, text, gesture and facial expressions. The problem with this data is that it will vary depending on the origin, culture, and nation. Because of this, it is difficult to detect human emotions more accurately. To solve this, our research makes use of electroencephalogram (EEG) signals that are directly collected from the brain. These signals not only ignore the external factors but also helps to detect real emotions arising from the brain. To conduct this research, a DEAP Dataset for emotion analysis using physiological signals is used. Firstly, the raw signal data is processed by removing the noise and converting the time series signal to statistical data. This statistical data is used to perform binary classification of four emotions valence, arousal, dominance, and liking. Various classifying techniques are examined to find the model that provides the best classification accuracy on this data. The experimental results show that Logistic Regression and Support Vector Machine are the best techniques for binary emotion classification with an accuracy of $69.25 \%$ and 70.35\% respectively.
\end{abstract}

Keywords - Emotion Analysis, EEG, Machine Learning, DEAP Dataset

\section{INTRODUCTION}

Emotions play a far greater role in determining business outcomes across industries than many executives grasp and experience. Emotional marketing become the new driver for business success. It refers to marketing and advertising efforts that primarily use emotion to make the audience notice, remember, share, and buy. Emotional marketing typically taps into a singular emotion, like happiness, sadness, anger, or fear, to elicit a consumer response [1]. Through years of studying emotions that can reach more than 34,000 distinct types, Dr. Robert Plutchik (psychologist) [2] in 2001 found that there are only eight primary emotions that serve as the foundation for all others: joy, sadness, acceptance, disgust, fear, anger, surprise and anticipation. Each primary emotion also has a polar opposite, so that:

Received: July 5, 2019

Reviewed: October 15, 2019

Accepted: October 29, 2019 
Joy is the opposite of sadness.

$>$ Fear is the opposite of anger.

$>$ Anticipation is the opposite of surprise.

$>$ Disgust is the opposite of trust.

Moreover, the combinations of these emotions has been described in Plutchik's recent wheel of emotions [3]. The wheel of emotion, however, is graph that can be used by human to understand emotion combinations based on color code that can specify emotion similarity, intensity or relations. This color classifications is meant for professionals to help them identifying and classifying emotions. Such wheel of emotions cannot be used by the machine learning technologies to classify emotions. However, in machine learning research, emotional analysis is regarded as an extension from the sentiment analysis. Sentiment analysis aims to classify texts (posts, sentences, or documents) into negative, positive or neutral [4]. Emotional analysis, on the other hand, is a more elaborated, deeper analysis of users' emotions that tries to inspect the psychology of different user behaviors revealing deeper human emotional connotations such as, anger, disgust, trust, sadness, joy, surprise, etc. Other machine learning approaches consider human facial images in recognizing the emotions and finding their intensity are vital [5]. However, the accuracy and the complexity of these two machine learning techniques points to lots of concerns on adopting them for accurately classifying emotions. Other more sophisticated machine learning approaches consider recognizing the emotions speech signals, body movement and physiological signals like skin resistance (SR) and electrocardiogram (ECG) are reporting more reliable accuracies but they are lacking the associated neurophysiological signals [6]. Therefore, physiological signals — especially EEG signals - become attractive because of the repeatability and objectivity for the estimation of emotion state. In recent years, with the development of sensor technology, it has become possible to monitor, record, and analyze multi-channel neurophysiological signals synchronously. Thus, many researchers have turned their attention to multi-channel EEG-based emotion recognition $[7,8]$. The case study in this paper describes a classification system that uses the electroencephalogram signals generated during the cognitive activity of the brain and to classify the emotions of the human at an instance of time. To develop a reliable methodology for emotion recognition, the first step is to perform an exploratory analysis which provides insights about the actual dimensions of the data and their correlation. Performing linear model analysis would define prediction accuracy on pre-processed data. This result helps us in understanding the signals and the structure. Feature extraction is performed, and the variables are encoded to perform the binary classification of the emotion of a participant. This can be applied in designing wearable gadgets to detect realtime human emotions, and it helps psychiatrists in treating psychological disorders like autism spectrum disorders (ASD), attention deficit hyperactivity disorder (ADHD) and anxiety disorders.

\section{RELATED RESEARCH WORK}

Niedermeyer and da Silva [9] classified the signals generated in the brain based on the intensity of the signal and the frequency range of the signal depending on the emotions associated with it. They defined that each type of signal or a range of signal has its own cognitive importance. The five frequency bands are Delta band $(1-4 \mathrm{~Hz})$, theta band $(4-$ $8 \mathrm{~Hz})$, alpha band $(8-12 \mathrm{~Hz})$, beta band $(13-25 \mathrm{~Hz})$ and gamma band $(>25 \mathrm{~Hz})$.

According to J. DellaBadia Jr [10], there are many ways to recognize the emotion, such as text, speech, facial expression, and brain waves. However, it is difficult to recognize emotion from some disabled people based on their appearance, such as autism. Facial expression may not depict the same emotion all the time; it might vary depending on the person, origin, and culture. Brain signal analysis is an alternative way to classify 
human emotion, which can be acquired by both invasive and non-invasive methods. An invasive method places the electrode on the exposed surface of a brain using surgical operations; this is not acceptable for most human beings and might be harmful to the participant. The non-invasive way is the way more comfortable and preferred way to collect the human brain signals in which electrodes are positioned on the scalp to record the brain activity. Magnetoencephalography (MEG), functional magnetic resonance imaging (fMRI), electroencephalogram (EEG) are methods to collect brain activity. Out of this EEG is most preferred and has advantages compared to other ones due to its high resolution and low cost of setup.

Several types of research have been conducted on the emotion recognition problem. K. Ishino et al. [11] proposed a system for feeling estimation through electroencephalogram data. The data used in the research is very small and are recorded randomly. Raw time series data has been used to analyse, and the data after Fast Fourier transformation and Wavelet Transformation has been feed into the neural network to distinguish different emotions and categorise the emotions into joy, sorrow, anger, and relaxation. A neural network with back propagation algorithm has been used as an accuracy of less than $50 \%$ is achieved.

Emotion recognition through speech has been conducted by Y. Chavhan [12]. In this research, three different language database is used to predict the emotion of a person. Signal pre-processing is used by applying pre-emphasis, framing and windowing techniques. Feature extraction is performed to find relevant features. Finally, the Support Vector Machine (SVM) classification has been used to classify the emotion. It has been observed that each language dataset has yielded different emotion for the same kind of data and the accuracy of the classification is different for different languages.

V.H. Ann [13] proposed an emotion recognition model based on Russell's circumplex model, Higuchi Fractal Dimension (HFD) algorithm and Support Vector Machine (SVM) as a classifier. In the first approach, machine learning was utilized for all EEG signals from numerous subjects while another used machine learning for each particular subject. The experimental results showed that the first approach is impossible to apply in practical applications because the EEG signal of each subject has the individual characteristic. Also, in the second, the model can recognize five basic states of human emotion with an average accuracy of $65.5 \%$.

R.Jenke [14] studied five different methods of feature extraction, and each method is evaluated with different machine learning models to find the optimum method of feature extraction. Results are presented concerning the performance of different feature selection methods, usage of selected feature types and selection of electrode locations. Features selected by multivariate methods slightly outperform univariate methods. Advanced feature extraction methods such as HOC, HOS, and HHS were found to outperform commonly used spectral power bands.

R.Nivedha [15] has proposed an EGG based emotion recognition using SVM and PCO in which human emotions are classified using EEG signals into four discrete states, namely happy, sad, angry and relaxed. Discrete wavelet transformation has been used for feature extraction, and the performance of the Support vector machine has been optimized by using particle swarm optimization algorithms. Model is able to generate good accuracy score when it is confined to only five electrodes readings.

\section{MACHINE LEARNING DATASETS AND ANALYTICS METHODS:}

For this case study we used the DEAP EEG dataset [16] that contains recording of the peripheral physiological signals of 32 participants each watched 40 one-minute long excerpts of music videos. Participants rated each video in emotional terms related to the 
levels of arousal, valence, like/dislike (on a scale of 1-9), dominance and familiarity. The DEAP dataset consists of two parts:

$>$ The ratings from an online self-assessment where 120 one-minute extracts of music videos were each rated by 14-16 volunteers based on arousal, valence, and dominance.

$>$ The participant ratings, physiological recordings and face video of an experiment where 32 volunteers watched a subset of 40 of the music videos. EEG and physiological signals were recorded, and each participant also rated the videos.

The dataset also consists of metadata files like online_ratings, video_list, participant_ratings,p articipant_questionnaire in CSV format and data_original, data_preprocessed are is .bdf and .dat file format. In this research, we make use of a data_preprocessed file which contains a separate .dat file for 32 participants, and each .dat file contains valence, arousal, dominance and liking ratings and the associated signal data of 40 trails of the participant. Table I illustrates the DEAP portion of the dataset considered in this case study.

Table I. DEAP Dataset Summary.

\begin{tabular}{|c|l|}
\hline \multicolumn{2}{|c|}{ DEAP Dataset Summary: The Online Subjective Annotation } \\
\hline Number of videos & 120 \\
\hline Video duration & 1 minute affective highlight \\
\hline Selection Method & 120 via last.fm and manually \\
\hline Number of ratings per video & $14-16$ \\
\hline Rating scales & Valence \\
& Arousal \\
& Dominance \\
& Liking \\
\hline Rating Values & The discrete scale of 1-9 \\
\hline \multicolumn{2}{|c|}{ Physiological Experiment } \\
\hline Number of participants & 32 \\
\hline Number of videos & 120 \\
\hline Number of trails & 40 \\
\hline Rating scales & Valence \\
& Arousal \\
& Dominance \\
& Liking \\
& Familiarity \\
\hline Rating Values & Familiarity : between 1 and 5 \\
& Others: float values between 1 and 9 \\
\hline Recorded Signals & 32 -Channel 512Hz EEG peripheral signals. \\
\hline
\end{tabular}

The following pre-processing steps have been done to the original 32 (.bdf) BioSemi format data signals which are recorded at $512 \mathrm{~Hz}$.

1. The data was down sampled to $128 \mathrm{~Hz}$.

2. EOG artefacts like eye blinking and noise are removed from the original signals.

3. A bandpass frequency filter from 4.0 to $45.0 \mathrm{~Hz}$ is applied to extract the signals that are in the frequency range of alpha, theta, beta, and gamma.

4. The datawere averaged to the joint reference by calculating the peak and base of the signal. 
5. The data was segmented into 60 second trials and a 3 second pre-trial baseline detached.

The data we obtain after preprocessing is the combined values of all the ratings in floating values of valence, arousal, dominance and liking with pure statistical values of the signals of all the 32 participants in 40 trails. Each participant has a separate .dat file which consists of two arrays one with labels valence, arousal, dominance and liking and other with the actual signal data for each trail. The pickled NumPy version of the data is in the form of a dictionary with labels as a nested array of keys and the data as a nested array of values. Each participant file has the following data (see Table II):

Table II. Structure of the .dat file.

\begin{tabular}{|l|l|l|}
\hline Array Name & Array Shape & Array Contents \\
\hline Labels & $40 \mathrm{X} 4$ & trail X label \\
\hline Data & $40 \mathrm{X} 40 \mathrm{X} 8064$ & trail X channel X data \\
\hline
\end{tabular}

The overall emotion classification process can be described in Figure 1.

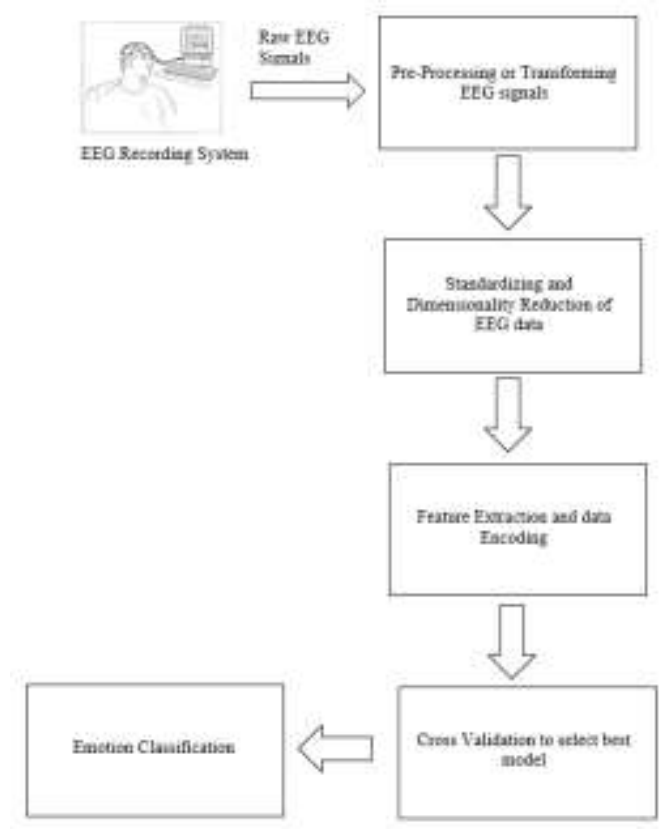

Fig. 1 Emotion Classification Architecture

The process of emotion classification starts by collecting the raw electroencephalogram (EEG) signals. This collected data is then pre-processed by removing artifacts and reducing the frequency range to $128 \mathrm{~Hz}$. The data is then normalized, and the dimensionality reduction is performed to make data ready for analysis. OneHotEncoding is performed on the normalized data to perform binary classification of each emotion such as valence, arousal, dominance, and liking. Multiple machine learning classification models are used to perform cross-validation to find the best model that classifies the emotion. After cross validation, it is found that Support Vector Machine (SVM) and Logistic Regression are the two best models to classify emotions. 
We implemented this architecture using Python, the Pre-processed python DEAP dataset has been used to perform emotion classification. The preprocessed dataset is in NumPy format, and the dataset consists of sampled signal data, and the ratings of each participant for each trail is recorded in the dataset. Each participant has a dat file with a total of 40 trails data (see Figure 2).

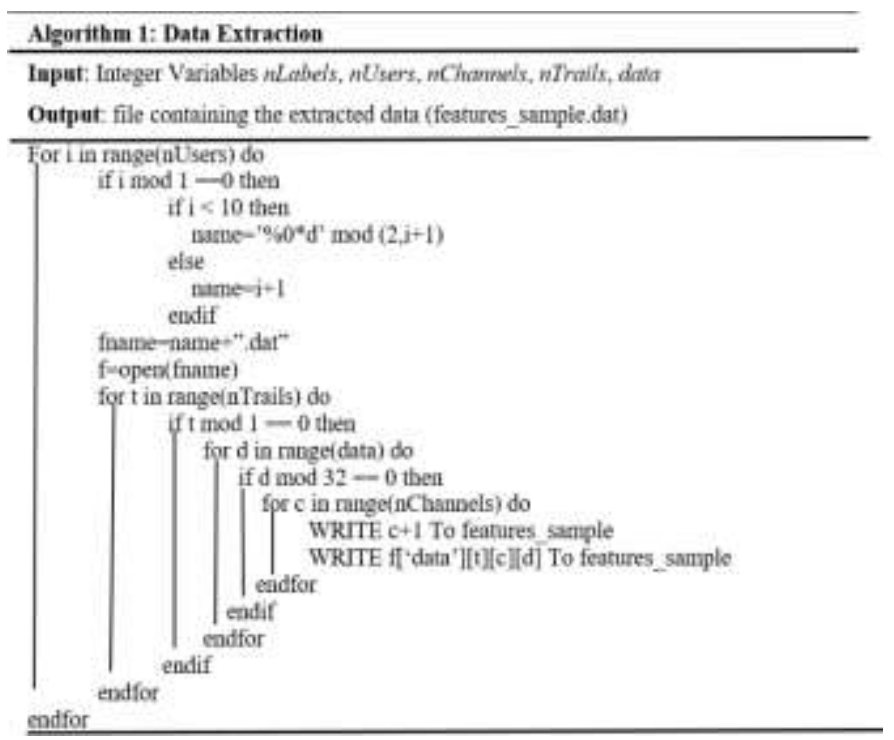

Fig. 2 Data Extraction Algorithm

Data extraction algorithm extracts the data and participant ratings from 32 .dat files and creates a single large file which contains the combined data of all the participants. The input the algorithm is the information about the size of the data like number of users, number of trails, number of channels, and size of the data which 32, 40, 32 and 8064 respectively.

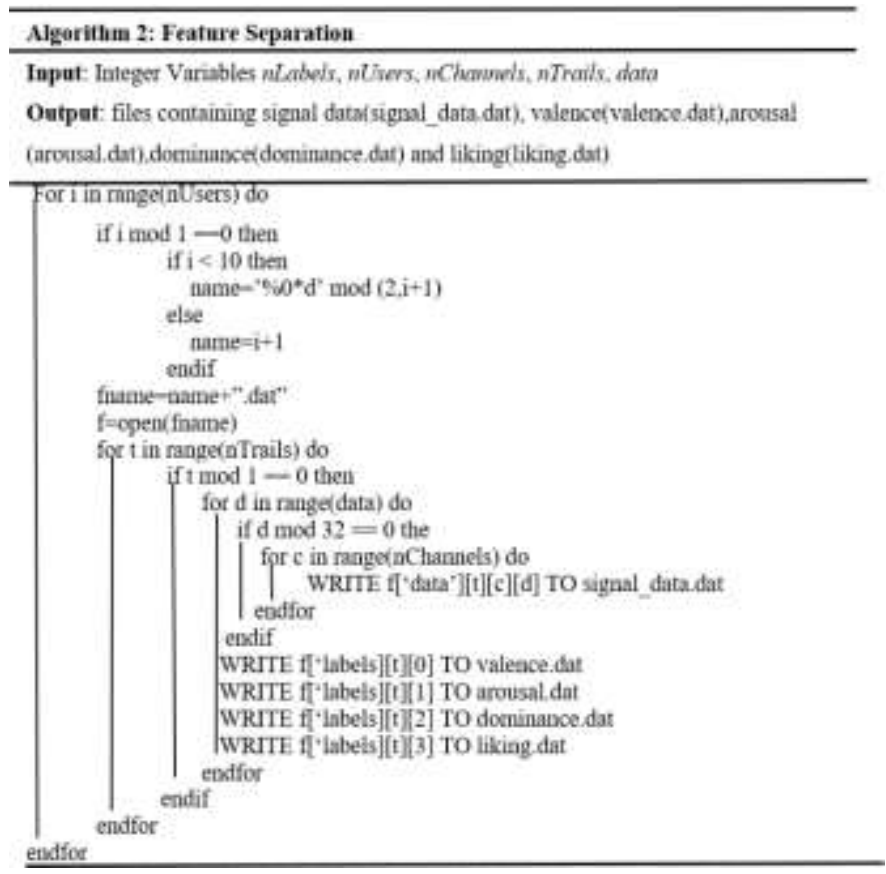

Fig. 3 Feature Separation 
Figure 3 illustrates our feature separation algorithm. The feature_sample.dat which is created in the data extraction process has all the data of all the participant in a single dat file. Feature separation algorithm separates the valence, arousal, dominance and liking rating of all the participant into four separate files valence.dat, arousal.dat, dominance.dat, and liking.dat. The actual signal data is stored in a separate file named signal_data.dat. Now, we have all the data required to test our models in a different file and we can focus on the encoding algorithm (see Figure 4).

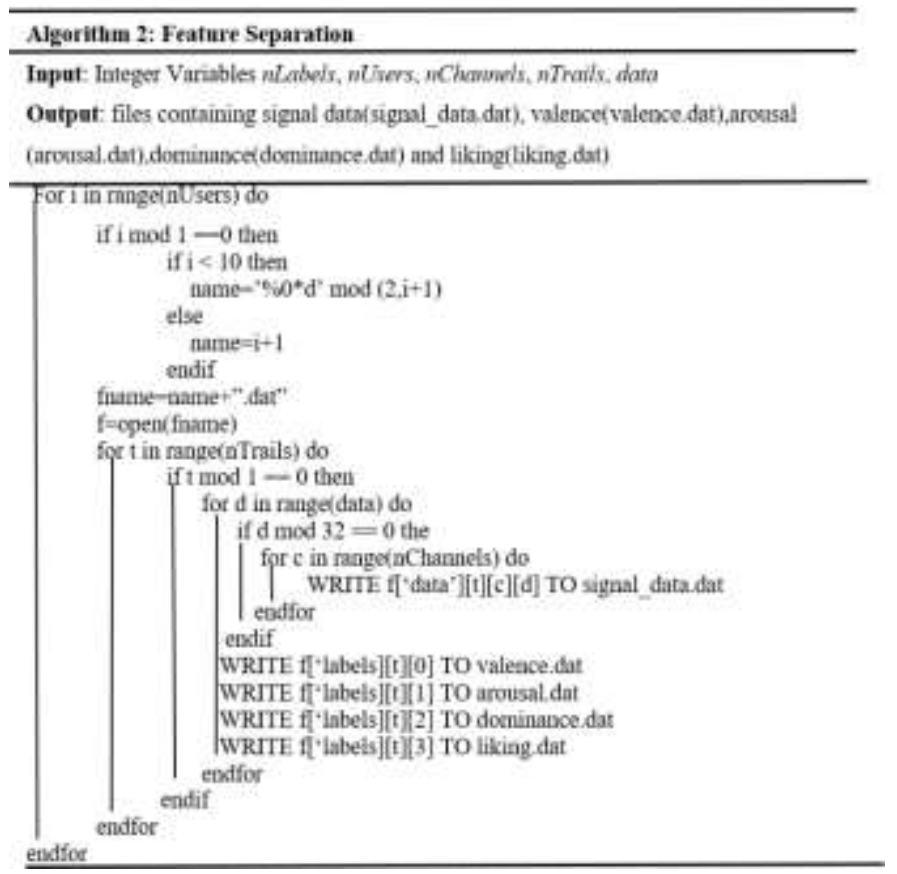

Fig. 4 Encoding Algorithm

Since, there is no co-relation between the four emotions or features valence, arousal, dominance, and liking. Binary classification of each emotion in correspondence to signal the need to be performed. To perform binary classification, we need to encode the ratings of each participant ratings into 0 or 1.Mean of the ratings which is 4.5 is chosen as the limit to encode the ratings into either class 0 or class. The values below are coded as class 1 with no valence, and the value greater than 4.5 is coded as class 0 with valence emotion. This encoding is performed on the three other file arousal.dat, dominance.dat, and liking.dat. 


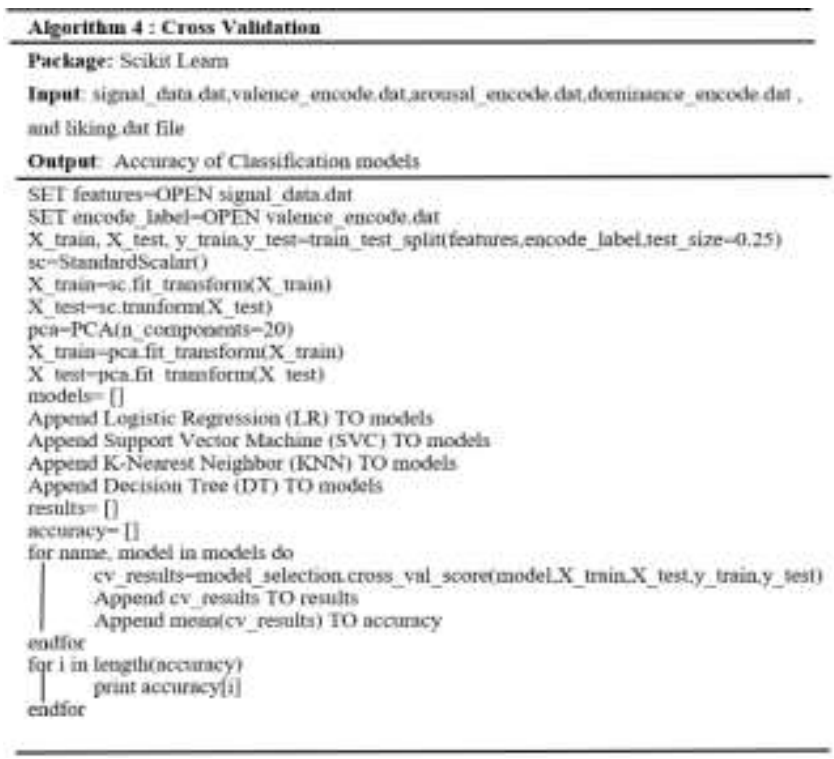

Fig. 5 Cross Validation Algorithm

Figure 5 illustrates the cross validation technique used to sample our data into different folds to check the accuracy of the different models on all the sampled data. Cross validation allows us to perform training and testing on the entire dataset by dividing and sampling into different folds for each iteration. In this experiment cross validation with five folds has been used. Classifying techniques like Logistic Regression (LR), Support Vector Machine (SVM), K-Nearest Neighbor are used to performing cross validation. Prior to cross validation standard scalar is applied on signal_data.dat file to standardize the data and Principle Component Analysis is performed to reduce the dimensionality of the signal data as this data is of high dimensionality. The same cross validation technique is performed separately with arousal, dominance, and like to find the model which yields high accuracy in binary classification of the emotion. The results yielded shows that Logistic Regression and Support Vector Machine is the best classification techniques to perform binary classifications of the emotions. The accuracy of each model with four emotions valence, arousal, dominance, and liking are explained in detail in the results section.

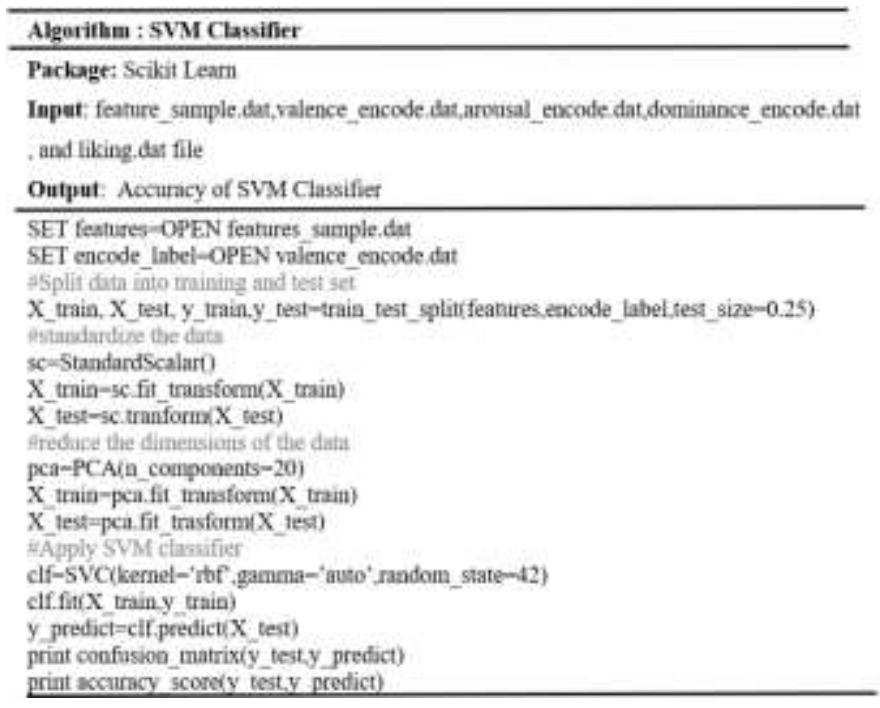

Fig. 6 Support Vector Machine 
Figure 6 illustrated the use of the Support Vector Machine with PCA and Standard scalar to classify the four emotions valence, arousal, dominance, and liking. The classification is performed on each emotion individually to classify it. Firstly, the data is split with a ratio of 0.75 and 0.25 into train and test set. Standard scalar and PCA are applied to the test and train data. An rbf kernel of support vector machine with a random state of 42 is used to define the classifier and prediction is performed on the test set. The confusion matrix is used to perform the model evaluation.

\section{EMOTIONS CLASSIFICATION ANALYTICS}

In this case study, a novel model and the best machine learning method is proposed to classify the human emotions using electroencephalogram data into one of the four classes' valence, arousal, dominance, and liking. A relatively large dataset of 32participants of 40 experiments a total of 1280 electroencephalogram (EEG) recordings have been used in the experiment-processing of the data is performed to convert the signals into statistical data. Preliminary analysis shows that there is no correlation between the four labels valence, arousal, dominance, and liking. One hot Encoding is performed four labels to enable binary classification of emotion. Dimensionality reduction technique Principal Component Analysis (PCA) is used to complement the feature extraction process, and data is standardized to perform model analysis on the processed signal data. Cross-validation is performed to find the model which yields the highest accuracy for this data. Logistic Regression and Support Vector Machine (SVM) outperformed K-Nearest Neighbor (KNN) and Decision trees with an average accuracy score of $69.25 \%$ and $70.35 \%$ respectively. Experiment results show that Logistic Regression (LR) and Support Vector Machine are the best model on this dataset to perform binary classification of the four emotions valence, arousal, dominance and liking successfully with an accuracy of $67.18 \%, 63.64 \%, 68.22 \%$ and $80.72 \%$ respectively with logistic regression and $66.66 \%, 63.64 \%, 68.22 \%$ and $79.68 \%$ for Support Vector Machine (SVM).The figure below shows the accuracy of all the models applied to the dataset (see Figures 7-10).

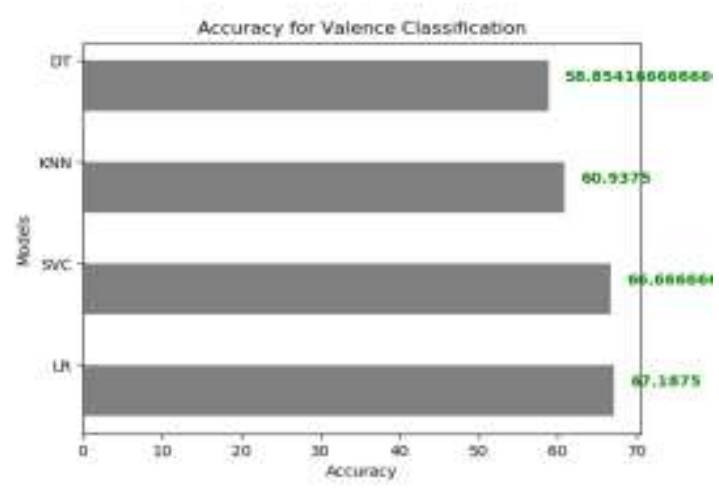

Fig. 7 Accuracy of Valence Classification 


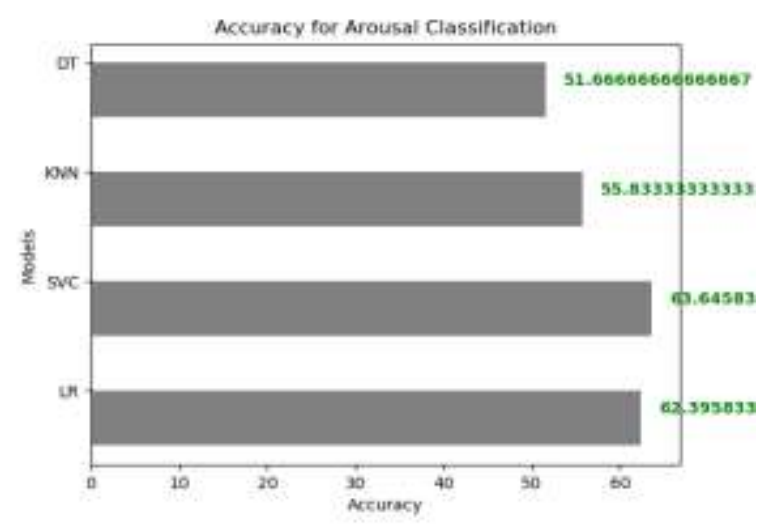

Fig. 8 Accuracy of Arousal Classification

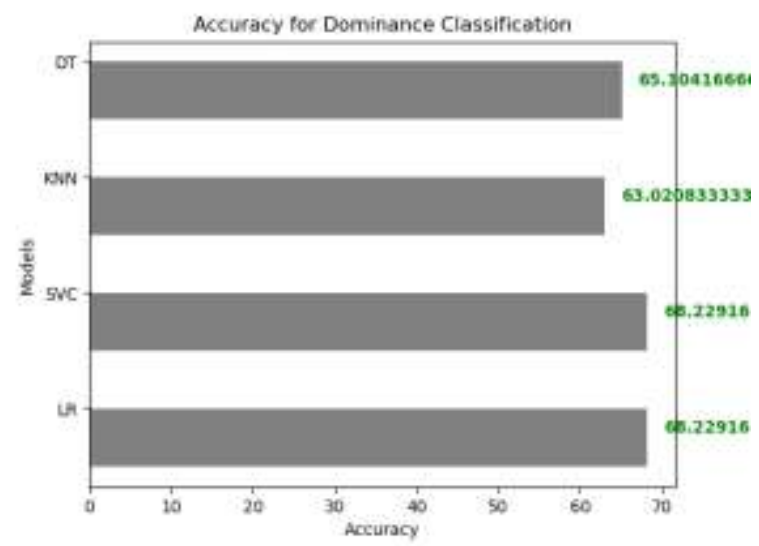

Fig. 9 Accuracy of Dominance Classification

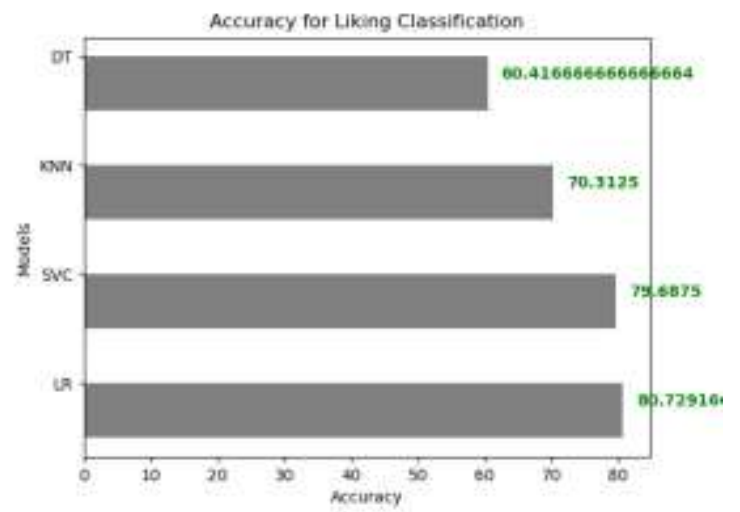

Fig. 10 Accuracy of Liking Classification

\section{CONCLUSIONS}

In this project an emotion classification using the preprocessed dataset for emotion analysis using EEG and physiological signal (DEAP) dataset is demonstrated successfully by classifying the emotion of a participant in terms of valence, arousal, dominance and liking with average accuracy of $69.25 \%$ and $70.35 \%$ with logistics regression and support vector machine respectively. Firstly, the preprocessed data has been extracted to separate the signal data, and the participant rating and then rating data is encoding to enable us to 
perform binary classification. Dimensionality reduction and standardization of data are performed before the application of classification models. Finally, Cross validation with five folds has been performed to find the best model that accurately classifies the emotion of a participant.

\section{ACKNOWLEDGMENTS}

This is a summary paper is part of the first author MSc Project research.

\section{REFERENCES}

[1] Allie Decker, The Ultimate Guide to Emotional Marketing, Hub Spot Blog, Originally published Aug 20, 2018 6:30:00 AM, updated August 08 2019, https://blog.hubspot.com/marketing/emotion-marketing

[2] R. Plutchik, "The Nature of Emotions Human emotions have deep evolutionary roots, a fact that may explain their complexity and provide tools for clinical practice," American Scientist, 89(4), pp.344-350, 2001.

[3] Melissa Donaldson, Plutchik's Wheel of Emotions, Available Online: https://www.6seconds.org/2017/04/27/plutchiks-model-of-emotions/

[4] Avetisyan H, Bruna O, Holub J. Overview of existing algorithms for emotion classification. Uncertainties in evaluations of accuracies. In Journal of Physics: Conference Series 2016 Nov (Vol. 772, No. 1, p. 012039). IOP Publishing.

[5] Mehta D, Siddiqui MF, Javaid AY. Recognition of emotion intensities using machine learning algorithms: A comparative study. Sensors. 2019 Jan;19(8):1897.

[6] Chao H, Dong L, Liu Y, Lu B. Emotion Recognition from Multiband EEG Signals Using CapsNet. Sensors. 2019 Jan;19(9):2212.

[7] Zheng WL, Dong BN, Lu BL. Multimodal emotion recognition using EEG and eye tracking data In2014 36th Annual International Conference of the IEEE Engineering in Medicine and Biology Society 2014 Aug 26 (pp. 5040-5043).

[8] Li Y, Huang J, Zhou H, Zhong N. Human emotion recognition with electroencephalographic multidimensional features by hybrid deep neural networks. Applied Sciences. 2017 Oct 13;7(10):1060.

[9] Lopes da Silva, Gonçalves, and de Munck (2009). "Electroencephalography (EEG). Encyclopedia of Neuroscience", 849-855.

[10] J. DellaBadia Jr., et al. "Assessment and cost comparison of sleep-deprived EEG, MRI, and PET in the prediction of surgical treatment for epilepsy, " Seizure, vol. 11, no. 5, pp. 303-309, 2002.

[11] K. Ishino and M. Hagiwara, "A Feeling Estimation System Using a Simple Electroencephalograph," Proc. ICSMC, vol.5, pp. 4204-4209, 2003.

[12] Y. Chavhan, M. Dhore, and P. Yesaware, "Speech Emotion Recognition using Support Vector Machine," International Journal of Computer Applications, vol. 1, no. 20, pp. 8-11, 2010. Available: 10.5120/431-636.

[13] V. H. Anh, M. N. Van, B. B. Ha, and T. H. Quyet, "A real-time model based Support Vector Machine for emotion recognition through EEG," 2012 International Conference on Control, Automation and Information Sciences (ICCAIS), 2012.

[14] R. Jenke, A. Peer, and M. Buss, "Feature Extraction and Selection for Emotion Recognition from EEG," IEEE Transactions on Affective Computing, vol. 5, no. 3, pp. 327-339, 2014.

[15] R. Nivedha, M. Brinda, D. Vasanth, M. Anvitha, and K. V. Suma, "EEG based emotion recognition using SVM and PSO," 2017 International Conference on Intelligent Computing, Instrumentation and Control Technologies (ICICICT), 2017.

[16] Koelstra S, Muhl C, Soleymani M, Lee JS, Yazdani A, Ebrahimi T, Pun T, Nijholt A, Patras I. Deap: A database for emotion analysis; using physiological signals. IEEE transactions on affective computing. 2011 Jun 9;3(1):18-31. 
International Journal of Disaster Recovery and Business Continuity Vol.10, November (2019) 